\title{
Continual Learning with Quasi-Newton Methods
}

\author{
Steven Vander Eeckt and Hugo Van hamme \\ KU Leuven \\ Department Electrical Engineering ESAT-PSI \\ Kasteelpark Arenberg 10, Bus 2441, B-3001 Leuven Belgium \\ \{steven.vandereeckt, hugo.vanhamme\}@esat.kuleuven.be
}

\begin{abstract}
Catastrophic forgetting remains a major issue when neural networks learn tasks sequentially. To alleviate catastrophic forgetting, Elastic Weight Consolidation (EWC) introduces a regularization loss, which, based on Bayesian inference, considers the posterior distribution over the previous tasks. However, when estimating the posterior with Laplace approximation, EWC approximates the Hessian by the diagonal of the Fisher information matrix, assuming the model parameters to be approximately independent. Very rarely will this be the case, and as such, the estimation of the Hessian and, consequently, of the posterior might be very poor. To overcome this, we propose Continual learning with Sampled Quasi-Newton (CSQN), which considers Quasi-Newton methods to obtain better Hessian approximations. Similar to EWC, CSQN uses these Hessian approximations to estimate the posterior and, as such, to encourage the model to update the model parameters in such a way that they remain optimal to both the current and previous tasks. Experimental results show that CSQN outperforms EWC as well as other baselines.
\end{abstract}

\section{INTRODUCTION}

Since the 2010s, in the so-called Deep Learning Revolution, Artificial Neural Networks (ANN) have been able to match or even surpass the performance of humans on a wide variety of tasks. Yet when presented with a set of tasks, to be learned sequentially - a setting which is referred to as Continual Learning (CL) - ANNs suffer from catastrophic forgetting [1]. Unlike humans, they seem unable to extend their knowledge without forgetting what they learned previously. Naively adapting the ANN to a new task generally leads to a deterioration of the performance of the network on previous tasks.

To overcome catastrophic forgetting, many CL methods have been proposed. One of the most well-known is Elastic Weight Consolidation (EWC) [2], which looks at CL from a Bayesian point of view. After training a task, it uses Laplace approximation [3] to estimate a posterior for the model parameters with respect to this task. When training a next task, this posterior is used by means of a regularization loss to prevent the model from catastrophically forgetting the previous task. To estimate the Hessian, which is needed in Laplace approximation to measure the (un)certainty of the model parameters, EWC uses the Fisher Information Matrix (FIM). Moreover, to simplify, EWC assumes that the FIM is approximately diagonal. Consequently, EWC uses the diagonal of the FIM to estimate the Hessian.

While EWC might often be very effective, its assumption of an approximately diagonal FIM (and, consequently, Hessian) will almost never hold. As a result, EWC's Hessian approximation might be poor and, consequently, so will be its approximation of the posterior, which could severely hurt EWC's performance in Continual Learning.

To improve EWC's approximation of the Hessian, we use Quasi-Newton (QN) methods [4]. QN methods, being secondorder optimization algorithms, involve (inverse) Hessian approximations which are updated at each iteration (but not computed explicitly) to find local optima. Two QN methods are BFGS (Broyden, Fletcher, Goldfarb, Shanno) and SR1 (Symmetric Rank-1), which apply a rank-2 and rank-1 update, respectively, to the Hessian approximation at each iteration. As we are only interested in QN methods because of their Hessian approximations, it would be disadvantageous would we have to train the model with the QN methods in order to obtain these approximations. Therefore, we propose to use Sampled Quasi-Newton (SQN) methods [5]. In SQN methods, the Hessian approximation is obtained by sampling around the current estimate for the local optimum. As such, the Hessian is re-computed at each iteration from scratch and is not based on previous iterations. Moreover, to further improve our method and to make it a literal extension to EWC, we use EWC's estimate of the Hessian, namely the diagonal of the FIM, as the initial approximation of the Hessian. Using SQN, we then improve this Hessian approximation, which is no longer diagonal. Similar to EWC, this improved Hessian approximation is used in the regularization loss to prevent forgetting of previously acquired knowledge when learning new tasks.

This paper is structured as follows. We start by reviewing related work in Section III. Next, we move on to Section III in which we explain our proposed method (Section III-D) after a short review of EWC (Section III-B) and (Sampled) QuasiNewton methods (Section III-C). In Section IV], we present the experimental results and compare our method to EWC and other baselines. Finally, we conclude the paper with Section V.

\section{RELATED WORK}

In general, there exist three large classes of CL methods: regularization-based methods, rehearsal-based methods and parameter-isolation methods [6].

Regularization-based. To prevent catastrophic forgetting, regularization-based methods regularize the learning of new tasks. Following [6], two classes of regularization-based methods are the prior-focused methods and data-focused methods. 
EWC, using the diagonal of the FIM to estimate the importance of parameters to previous tasks, belongs to the priorfocused methods. Similar to EWC are Synaptic Intelligence (SI) [7] and Memory-Aware Synapses (MAS) [8], which compute the importance weights of the model parameters differently. While SI estimates the importance of parameters by accumulating each parameter's contribution to the reduction in the loss, MAS considers the gradients of the squared norm of the model's output with respect to each parameter. Similar work includes [9], [10], [11].

Many works have built on EWC's ideas. Online EWC [12] allows EWC to be used in an online way, without requiring task boundaries. [13] proposes Riemanian Walk, which combines the regularization losses of Online EWC and SI. Moreover, to address EWC's assumption of an approximately diagonal FIM, R-EWC (Rotated-EWC) [14] rotates the parameter space layer-wise such that the corresponding FIM is indeed approximately diagonal. While R-EWC improves the performance of EWC when adapting one task to another, REWC only applies the reparametrization to the previous task and has no mechanism to combine the importance weights of all tasks learned so far. Meanwhile, [15] proposes to use Kronecker factored approximations of the FIM [16], which allows to compute and store the FIM efficiently for each layer, thus obtaining a block diagonal approximation of the FIM. As such, unlike EWC, they consider interactions between model parameters within the same layer, but not across layers.

A second class of regularization-based methods are the datafocused methods, whose main idea is knowledge distillation [17], to transfer knowledge from the previous to the new model. Examples include Learning Without Forgetting (LWF) [18], but also [19], [20].

Instead of using a regularization loss, other methods constrain the gradients when learning new tasks. This includes Orthogonal Gradient Descent (OGD) [21], which updates the gradient used in Stochastic Gradient Descent (SGD) such that it is orthogonal to the gradients of previous tasks; Gradient Projection Memory (GPM) [22], which partitions the gradient space into two orthogonal subspaces, forcing the gradient to lie in the subspace least harmful to previous tasks; and the work of [23], [24].

Rehearsal-based. Rehearsal-based methods assume access to a memory of representative samples of previous tasks. Examples include [25], [26]. Gradient Episodic Memory (GEM) [27] can be considered an overlap between rehearsal-based and regularization-based methods [28], as it introduces a regularization loss based on a set of representative samples, assuring SGD's gradient updates do not increase the loss of the representative samples. GEM has been extended in [29], [30]. Similar to GEM is [31].

Within the rehearsal-based methods, the pseudo-rehearsal methods, instead of requiring access to a memory of representative samples, train a generator to simulate data of previous tasks. Examples include [32], [33], [34].

Parameter-isolation. Parameter-isolation methods expand the network when tasks are added, to avoid interference between tasks. Examples include [35], [36], [37], [38], [12]. The latter proposes Progress \& Compress (P\&C) [12], which uses online EWC in the 'progress' stage. Another method on the intersection between parameter-isolation and regularizationbased methods is AR1 [28], which combines Copy Weight with Reinit (CWR) [39] with SI and EWC.

\section{Continual LeARning With SAMPLED QUASI-NEWTON}

In this section, we explain our proposed CL method. However, we start this section by the problem formulation, followed a review of EWC and (Sampled) Quasi-Newton methods.

\section{A. Problem Formulation}

We have a sequence of $T$ tasks represented by data $D_{1}, D_{2}, \ldots, D_{T}$ with, for $(x, y) \in D_{i}, x \in \mathbb{R}^{d}$ is the input to the model and $y$ the class label. The tasks are learned in sequence and, during training of any task $t$, the model has access to $D_{t}$ only. The goal is to learn task $t$ without forgetting the knowledge extracted from $D_{1}, D_{2} \ldots, D_{t-1}$, i.e. if $\theta \in \mathbb{R}^{N}$ are the model's parameters, and $\theta^{(t)}$ are the parameters after learning the first $t$ tasks in sequence, then we seek: $\theta^{(T)}=\arg \max _{\theta} P\left(\theta \mid D_{1}, D_{2}, \ldots, D_{T}\right)$ where $\theta^{(t)}$ is obtained by training $\theta^{(t-1)}$ on $D_{t}$ without access to $D_{1}, D_{2}, \ldots, D_{t-1}$.

Moreover, denote by $f_{\theta}(\cdot)$ the model with parameters $\theta$. $p_{\theta}(y \mid x)$ is its softmax output for class $y$ given input $x$, while $L_{t}(\theta)$ is its cross-entropy loss on task $t$.

\section{B. Elastic Weight Consolidation}

EWC looks at CL from a Bayesian point of view. When learning a second task, the optimal parameters $\theta^{(2)}$ can be found as follows:

$$
\begin{aligned}
\theta^{(2)} & =\underset{\theta}{\arg \max } \log P\left(\theta \mid D_{1}, D_{2}\right) \\
& =\underset{\theta}{\arg \max } \log P\left(D_{2} \mid \theta\right)+\log P\left(\theta \mid D_{1}\right)
\end{aligned}
$$

This result is obtained by applying Bayes' rule to $P\left(\theta \mid D_{1}, D_{2}\right)$ assuming that $D_{1}$ and $D_{2}$ are independent [2]. The first term on the right hand side of Equation 1 corresponds to optimizing the cross-entropy loss for $D_{2}$, i.e. $L_{2}(\theta)$; the second term, the posterior after training on task 1 , is intractable. However, it can be estimated using Laplace approximation [3]:

$$
\log P\left(\theta \mid D_{1}\right)=-\frac{\lambda}{2}\left(\theta-\theta^{(1)}\right)^{T} \Lambda\left(\theta-\theta^{(1)}\right)
$$

In Equation 2. $\Lambda$ is the Hessian of $L_{1}\left(\theta^{(1)}\right)$. Laplace approximation thus approximates the posterior $P\left(\theta \mid D_{1}\right)$ by a normal distribution $N\left(\theta^{(1)}, \Lambda^{-1}\right)$. To approximate the Hessian $\Lambda$, EWC considers the FIM, which is defined as:

$$
F_{\theta}=\mathbb{E}_{(x, y) \sim D}\left[\left(\frac{\partial \log p_{\theta}(y \mid x)}{\partial \theta}\right)\left(\frac{\partial \log p_{\theta}(y \mid x)}{\partial \theta}\right)^{T}\right]
$$

To simplify, moreover, EWC approximates the FIM by its diagonal. Combining Equations 1, 2 and the assumption that the FIM is approximately diagonal, the loss while training task 2 becomes the following:

$$
\operatorname{Loss}_{2}(\theta)=L_{2}(\theta)+\frac{\lambda}{2} \sum_{i=1}^{N} \Omega_{i}^{(1)}\left(\theta_{i}-\theta_{i}^{(1)}\right)^{2}
$$


with $\Omega^{(1)} \in \mathbb{R}^{N}$ the diagonal of $F_{\theta^{(1)}}, \theta_{i}$ the $i$ th parameter of $f_{\theta}(\cdot)$ and $\lambda$ a hyper-parameter, determining the weight of the regularization. $\Omega_{i}^{(1)}$ can be seen as an estimate of how important $\theta_{i}^{(1)}$ is to $f_{\theta^{(1)}}$ with respect to task 1 .

To extend EWC to more than two tasks, a regularization loss can be introduced for each term. However, [40] suggests combining these regularization losses in one term. When learning task $t$, one then obtains:

$$
\operatorname{Loss}_{t}(\theta)=L_{t}(\theta)+\frac{\lambda}{2} \sum_{i=1}^{N}\left(\sum_{j=1}^{t-1} \Omega_{i}^{(j)}\right)\left(\theta_{i}-\theta_{i}^{(t-1)}\right)^{2}
$$

As such, it suffices to store $\sum_{j=1}^{t-1} \Omega^{(j)}$ and $\theta^{(t-1)}$ in order to learn task $t$ with EWC.

While EWC might often alleviate catastrophic forgetting, its assumption that the FIM is approximately diagonal will almost never hold. On the contrary, in many cases the diagonal of the FIM might often capture but a small amount of the total weight. As such, EWC's Hessian approximation might be poor, and, consequently, so will be its approximation to the posterior. By considering better Hessian approximations, we can improve the approximations to the posterior and, therefore, the performance on continual learning.

\section{C. (Sampled) Quasi-Newton Methods}

To improve EWC's Hessian approximation, QN methods can be used. However, let us first start by reviewing QN and SQN methods.

Quasi-Newton. Given a function $f(\cdot)$ to be optimized with respect to $x \in \mathbb{R}^{n}$. Let $f_{k}=f\left(x_{k}\right)$ with $x_{k}$ the current solution at iteration $k$ and $\nabla f_{k}$ its gradient. In order to iteratively optimize $f$, QN methods consider the quadratic model:

$$
m_{k}(p)=f_{k}+\nabla f_{k}^{T} p+\frac{1}{2} p^{T} B_{k} p
$$

with $B_{k}$ an estimate of the Hessian at the current solution $x_{k}$. QN methods move to the next iteration by taking a step in the direction of the quadratic model's minimizer, $p^{*}=-B_{k}^{-1} \nabla f_{k}$. Next, the Hessian estimate is updated and the process is repeated until convergence.

QN methods differ in how this Hessian estimate is computed. Two well-known QN methods are BFGS and SR1, which respectively apply a rank-two and rank-one update to the Hessian estimate at each iteration. For BFGS, the Hessian estimate at iteration $k$ is computed as follows [4]:

$$
B_{k}=B_{0}-\left[\begin{array}{ll}
B_{0} S_{k} & Y_{k}
\end{array}\right]\left[\begin{array}{cc}
S_{k}^{T} B_{0} S_{k} & L_{k} \\
L_{k}^{T} & -D_{k}
\end{array}\right]^{-1}\left[\begin{array}{c}
S_{k}^{T} B_{0} \\
Y_{k}^{T}
\end{array}\right]
$$

with $S_{k}=\left[s_{0}, s_{1} \ldots, s_{k-1}\right]$ and $Y_{k}=\left[y_{0}, y_{1} \ldots, y_{k-1}\right]$ where $s_{i}=x_{i+1}-x_{i}$ and $y_{i}=\nabla f_{i+1}-\nabla f_{i}$. Moreover, $D_{k}$ is diagonal matrix with $\left(D_{k}\right)_{i, i}=s_{i}^{T} y_{i}$ and $L_{k}$ is a lower diagonal matrix whose element $\left(L_{k}\right)_{i, j}=s_{i}^{T} y_{j}$ only when $i>j$, else it is zero. Finally, $B_{0}$ is an initial estimate for the Hessian, commonly $B_{0}=\gamma I$ with $I$ the $n \times n$ identity matrix. Similarly, for SR-1, the Hessian estimate at iteration $k$ can be computed as follows [4]:

$B_{k}=B_{0}+\left(Y_{k}-B_{0} S_{k}\right)\left(D_{k}+L_{k}+L_{k}^{T}-S_{k}^{T} B_{0} S_{k}\right)^{-1}\left(Y_{k}-B_{0} S_{k}\right)^{T}$
While it is not guaranteed that the Hessian estimates produced by SR1 are positive definite, SR1 has proven to yield better Hessian estimates than BFGS [4].

Furthermore, to limit the memory requirements of the QN methods, only the $M$ most recent vectors are stored in $S_{k}$ and $Y_{k}$.

Sampled Quasi-Newton. To further improve QN methods, [5] proposes Sampled Quasi-Newton methods. While in QN methods $S$ and $Y$ depend on $(s, y)$ pairs from the current iteration and previous $M-1$ iterations, SQN methods "forget the past" and, at each iteration, build the $S$ and $Y$ matrices from scratch [5]. As shown in [5], this yields better Hessian approximations. Moreover, as $S$ and $Y$ are obtained from scratch at each iteration, it does not take $M$ iterations until $S$ and $Y$ contain $M$ components, which is the case for QN methods. Once $S$ and $Y$ are sampled, SQN proceed in the same way as QN methods.

To obtain $S_{k}$ and $Y_{k}$ at iteration $k$ from scratch, SQN methods sample points $\tilde{x}_{i} \in \mathbb{R}^{n}$ with $i=1, . ., M$ in the neighbourhood of $x_{k}$. Then, they compute $s_{i}=x_{k}-\tilde{x}_{i}$ and $y_{i}=\nabla f\left(x_{k}\right)-\nabla f\left(\tilde{x}_{i}\right)$ and, similarly to QN methods, store these in $S_{k}=\left[s_{1}, s_{2}, \ldots, s_{M}\right]$ and $Y_{k}=\left[y_{1}, y_{2}, \ldots, y_{M}\right]$.

The process of obtaining $S$ and $Y$ by sampling is summarized in Algorithm 11. Note that here, $\tilde{x}_{i}$ is sampled from $N\left(x_{k}, \Sigma\right)$ with $\Sigma$ a diagonal matrix. Moreover, note that $y$ is computed using $\nabla^{2} f\left(x_{k}\right) s$ instead of $y=\nabla f_{k}-\nabla f\left(\tilde{x}_{i}\right)$. The advantage of the former case is that it is invariant to the scale of $\Sigma$, whereas in the latter case the scale of $\Sigma$ must be carefully chosen.

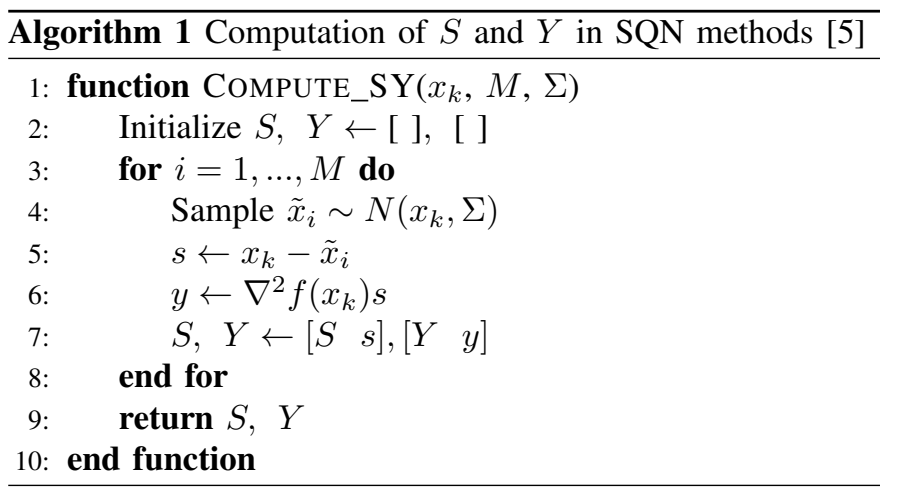

To assure that the Hessian approximations for BFGS are positive definite, an $(s, y)$ pair may only be added to $S$ and $Y$ if $s^{T} y>\kappa\|s\|^{2}$ [5]. On the other hand, in order for Equation 8 to be well-defined for SR1, $\left|s^{T}(y-B s)\right| \geq\left.\kappa|| s\right|^{2}$ must hold for all $(s, y)$ pairs [5]. Therefore, only $(s, y)$ pairs which satisfy the required condition are added to $S$ and $Y$. Note that this occasionally may result in $S$ and $Y$ containing less than $M$ components. However, [5] shows that, if $\kappa$ is sufficiently small (e.g. $\kappa=1 e-8$ ), the condition is extremely rarely violated.

\section{CSQN}

Let us now combine EWC and SQN into our proposed CL method: Continual learning with Sampled Quasi-Newton (CSQN).

Given Equation 2, we consider SQN methods to find an estimate of the Hessian of $L_{1}\left(\theta^{(1)}\right)$, which, unlike in EWC, 
does not assume the model parameters to be independent. As SQN methods obtain $S$ and $Y$ at each iteration from scratch, Hessian approximation can be obtained without having to optimize the ANN with a (S)QN method. Given $\theta^{(1)}$, we can thus use Algorithm 1 to sample $S^{(1)}$ and $Y^{(1)}$. Next, Equations 7 and 8 return an estimate of the Hessian, $B^{(1)}$, which can then be used in Equation 2, resulting in the following loss function for training the second task:

$$
\operatorname{Loss}_{2}(\theta)=L_{2}(\theta)+\frac{\lambda}{2}\left(\theta-\theta^{(1)}\right)^{T} B^{(1)}\left(\theta-\theta^{(1)}\right)
$$

Dropping the superscripts for convenience, note that $B$ can be computed from $S, Y$ and $B_{0}$, hence, it suffices to store these three objects, which combined are $2 M+1$ vectors of size $N$.

Moreover, as SR1 with $M(s, y)$ pairs results in a rank- $M$ (without the initial Hessian estimate $B_{0}$ ) approximation of the Hessian, it should suffice to store $M+1$ instead of $2 M+1$ vectors. To this end, we compute $X \in \mathbb{R}^{N \times M}$ and $A \in \mathbb{R}^{N \times N}$ such that we can write Equation 8 as $B=B_{0}+X A^{-1} X^{T}$. Next, we apply the Cholesky factorization to $A^{-1}$, such that $A^{-1}=L L^{T}$, which allows us to write $B=B_{0}+Z Z^{T}$ with $Z=X L$. However, the Cholesky factorization requires $A$ to be positive definite. If $A$ is not positive definite, which may be problematic because in that case the regularization loss of Equation 9 might be negative, we can compute the Eigenvalue Decomposition (EVD) of $A^{-1}: A^{-1}=V \Gamma V^{T}$. Next, we obtain $\Gamma^{\prime}$ by setting the negative eigenvalues in $\Gamma$ to zero, after which we compute the QR-factorization of $\left(V \sqrt{\Gamma^{\prime}}\right)^{T}$ such that $A^{-1} \approx V \Gamma^{\prime} V^{T}=(Q R)^{T} Q R=R^{T} R=L L^{T}$ with $L=R^{T}$. As such, we find $Z=X L$ and it suffices to store $Z \in \mathbb{R}^{N \times M}$ and $B_{0} \in \mathbb{R}^{N}$. Moreover, it is now assured that the regularization loss for CSQN with SR1 will always be positive.

Algorithm 2 summarizes the procedure of obtaining $Z$ from $S$ and $Y$.

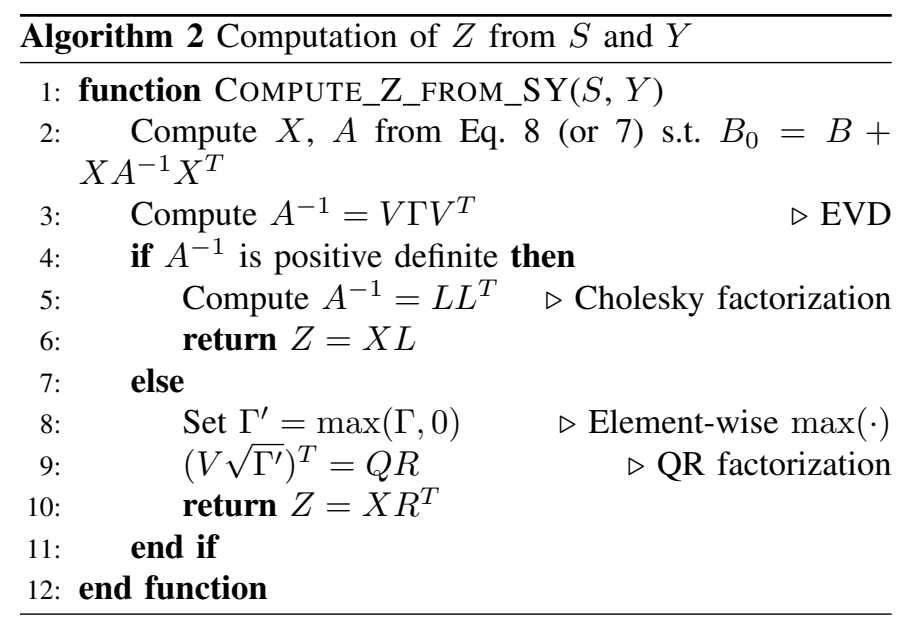

To further improve CSQN, we use $\Omega^{(1)}$, the diagonal of the FIM, in two ways. First, as covariance matrix $\Sigma$ to compute $S$ and $Y$ (see Algorithm 1 , we set $\Sigma=\left(\Omega^{(1)}+\epsilon \max \left(\Omega^{(1)}\right) I\right)^{-1}$ with $\max \left(\Omega^{(1)}\right)$ the maximum of $\Omega^{(1)}$ and $I$ the $N \times N$ identity matrix. The idea is that knowing the Hessian would lead to better sampling, and the diagonal of the FIM is an initial estimate of the Hessian. This has proven to work well in our experiments. However, note that this introduces a new hyper-parameter $\epsilon$ to avoid numerical problems when some elements of $\Omega^{(1)}$ are (close to) zero. Second, we take $\Omega^{(1)}$ as our initial estimate of the Hessian, i.e. $B_{0}^{(1)}=\Omega^{(1)}$. This means that the regularization loss of CSQN can be split into two terms: one term is related to $B_{0}$ and therefore equal to EWC's regularization loss, while the second term is related to $S$ and $Y$ and the corresponding rank- $2 M$ or rank- $M$ Hessian approximations. As such, CSQN literally extends EWC.

Moreover, note that Algorithm 1 requires computing the gradient of $L_{1}\left(\theta^{(1)}\right)$, which is also required to compute $\Omega^{(1)}$. Therefore, computing $\Omega^{(1)}$ does not entail an extra forward or backward pass over the task's data.

To adapt a model trained on $t-1$ tasks to a $t$ th task, we follow the reasoning of [40] and consider the following loss:

$\operatorname{Loss}_{t}(\theta)=L_{t}(\theta)+\frac{\lambda}{2}\left(\theta-\theta^{(t-1)}\right)^{T}\left(\sum_{j=1}^{t-1} B^{(j)}\right)\left(\theta-\theta^{(t-1)}\right)$

A disadvantage of our method as currently proposed is that the memory requirements increase linearly with the number of tasks. To overcome this, a straightforward way would be to use Algorithm 2 to compute $Z^{(i)}$ for each task $i$, after which these matrices could be concatenated into one large matrix $Z^{(\leq t)}$, to which we can then apply Singular Value Decomposition (SVD) to reduce its number of columns to e.g. $M$ (SR1) or $2 M$ (BFGS). This could be done after each task, and as such the number of columns $Z^{(\leq t)}$ could be kept constant (at $M$ or $2 M)$ as $t$ increases. Moreover, to compensate for the reduction of regularization loss (as we reduce the number of columns of $Z^{(\leq t)}$ from $M_{b}$ to $M_{a}$ ), we multiply the reduced $\tilde{Z}^{(\leq t)}$ by $\sqrt{M_{b}} / \sqrt{M_{a}}$. We call this strategy $C T$.

One problem of this strategy might be that at task $t+1$, when $\tilde{Z}^{(\leq t)}$ and $Z^{(t+1)}$ are concatenated and reduced with SVD, $\tilde{Z}^{(\leq t)}$ represents $t$ times more tasks than $Z^{(t+1)}$, yet both contain the same number of columns. Consequently, one can expect $\tilde{Z}^{(\leq t+1)}$ to contain less and less information about older tasks as $t$ increases. To overcome this, we could give the columns of $\tilde{Z}^{(\leq t)}$ a weight of $\sqrt{t}$ before applying SVD. We refer to this strategy as CT-W. Another option would be to group the tasks into a binary tree. All tasks start at the bottom of the tree and are grouped two-by-two. Once a task and its neighbour in the tree are both learned by the model, their $Z$ matrices are concatenated and reduced with SVD into a $Z$ matrix of size $M$ for SR1 and $2 M$ for BFGS. The resulting matrix moves up the tree and the process is repeated. As such, unlike in the CT strategy, any two $Z$ matrices that are concatenated and reduced represent an equal number of tasks. With this strategy, which we refer to as BTREE, the number of components to store is not constant, but increases with only $\log _{2}(T)$.

Algorithm 3 summarizes our method, CSQN. Note that here, reduce $(\cdot)$ could be any user-defined function to reduce $Z^{(\leq t)}$. If it is defined, or if it is not (because reducing the number of components is not desired) but the considered QN method is SR1, then Algorithm 2 is used to compute $Z^{(t)}$ (Line 10. In the former case, by applying reduce $(\cdot)$ to $Z^{(t)}$ and $\tilde{Z}^{(<t)}$, 
$\tilde{Z}^{(\leq t)}$ is obtained (Line 14 ), which is used in the regularization loss of Line 4 when learning task $t+1$. In the latter case, on the other hand, $Z^{(t)}$ is stored along $Z^{(1)}, \ldots, Z^{(t-1)}$, which are all required to compute the regularization loss for task $t+1$. Finally, if it is not desired to reduce the number of components and the considered method is BFGS, then it is not required to compute $Z^{(t)}$, and it suffices to store $S^{(t)}$ and $Y^{(t)}$. To learn task $t+1$, then, $S^{(1)}, \ldots, S^{(t)}, Y^{(1)}, \ldots, Y^{(t)}$ and Equation 7 are used.

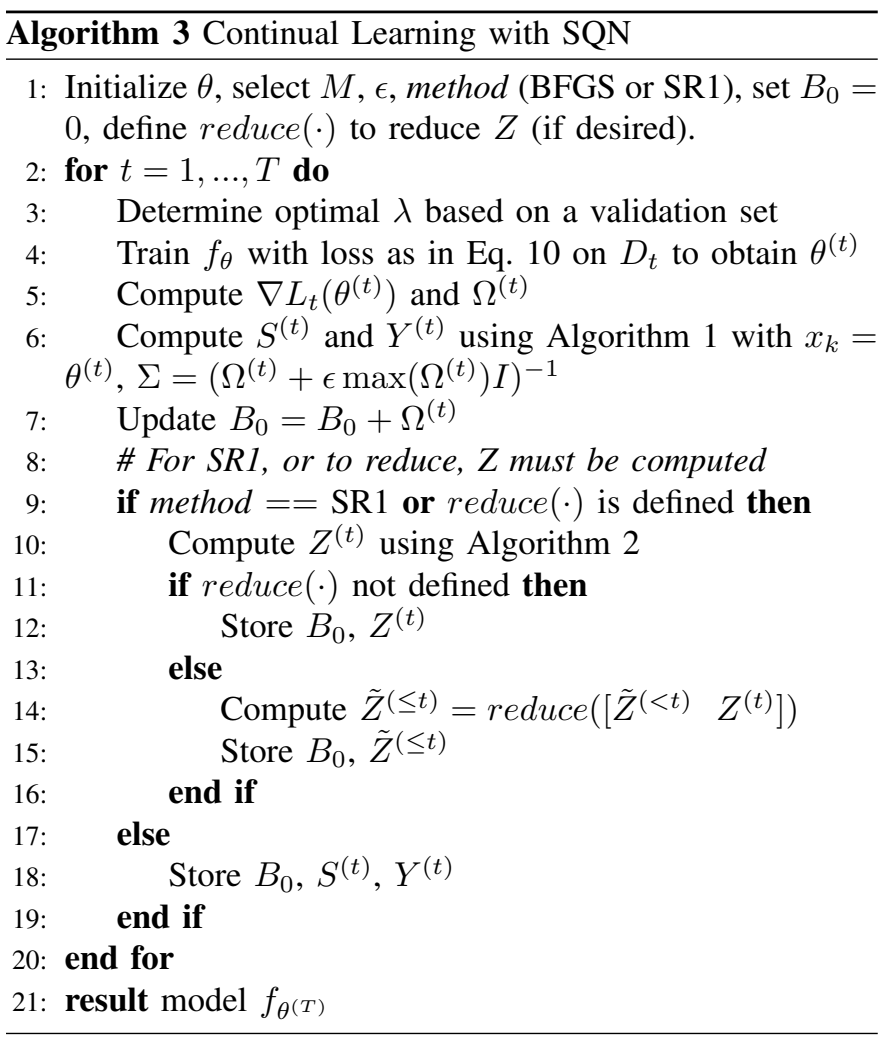

\section{EXPERIMENTS}

Datasets. We consider the Rotated MNIST [27], Split CIFAR-10/100 [7] and Vision Datasets [15] benchmarks. In the Rotated MNIST benchmark, similar to [21], the MNIST [41] images for task $t$ are rotated by an angle of $\gamma(t-1)$. To obtain a validation set, we split the MNIST training data into 55,000 training and 5,000 validation images. For the Split CIFAR-10/100 experiments, we split the CIFAR-100 data [42] into $T$ subsets, each with $100 / T$ non-overlapping classes. As the first task in this benchmark is the CIFAR-10 dataset, we thus have $T+1$ tasks. We split the CIFAR-10 and CIFAR-100 training sets into 45,000 training and 5,000 validation images. Finally, in the Vision Datasets benchmark, the tasks are five vision datasets: MNIST, CIFAR-10, SVHN [43], FashionMNIST [44] and notMNIST [45]. Again, we split the training data for each dataset into $95 \%$ training and $5 \%$ validation images. In the Rotated MNIST experiments, the entire model is shared, while in the Split CIFAR-10/100 and Vision Datasets benchmarks, the classification layer is taskspecific.

Baselines. The baselines are: (a) EWC, as explained in III-B, (b) Memory-Aware Synapses (MAS) [8]: similar to
EWC but computes $\Omega^{(t)}$ differently, without requiring the class labels; (c) Learning without Forgetting (LWF) [18]: uses knowledge distillation between $f_{\theta^{(t)}}$ and $f_{\theta}$ (the current model) on data from task $t+1$ when learning task $t+1$; (d) Kronecker-Factored approximated Laplace (KF) [15]: uses Kronecker-factored approximations of the FIM to estimate the posterior with Laplace approximation; (e) Fine-Tuning: naïvely adapts the model to new tasks without using any CL algorithm - considered the lower bound.

Evaluation Metrics. To evaluate the performance of the methods, we consider the average accuracy (ACC) and backward transfer (BWT), as in [27]. The latter was defined as follows, assuming that $R_{i j}$ is the accuracy on task $j$ after learning task $i$ and that $T$ tasks are learned:

$$
\mathrm{BWT}=\frac{1}{T-1} \sum_{i=1}^{T-1} R_{T i}-R_{i i}
$$

Consequently, negative BWT indicates forgetting.

Models and training. For the MNIST benchmarks, we consider an MLP with two hidden layers of 256 neurons and ReLU activations and a dropout [46] of $25 \%$. We train the model for 10 epochs for each task with Adam $\left(\beta_{1}=0.9, \beta_{2}=\right.$ 0.999 ) with a learning rate of 0.001 . For the Split CIFAR10/100 experiments, we considered the 'simple' Resnet-32 that was used in [47] on the CIFAR-10 dataset, as it was implemented by [48]. The first task (the CIFAR-10 dataset) was trained for 15 epochs, while the subsequent Split CIFAR100 tasks were trained for 10 epochs. The optimizer was the same as for the MNIST experiments. Finally, for the Vision Datasets experiments, we used a similar LeNet [49] as in [15], with two convolutional layers with convolutions of $5 \times 5$ and 20 and 50 channels, respectively, followed by a fullyconnected layer with 500 neurons. However, we added another fully-connected layer of 84 neurons, to reduce the number of neurons in the classification layer, which is task-specific and therefore not subject to regularization. Each of the five tasks is trained for 50 epochs using the same optimizers as for the MNIST experiments.

Implementation. For our method, CSQN, we considered $\epsilon=1 e-4$ and $\kappa=1 e-8$ in all experiments. Moreover, in all experiments, we try CSQN with both BFGS (referred to as CSQN-B) and SR1 (CSQN-S) with $M=10$ and $M=20$ (reported between brackets). For the hyper-parameter selection with respect to $\lambda$, the regularization weight, for both our method and the baselines, as well as for the other hyperparameters for the baselines, see the appendix. All experiments were done in PyTorch [50]. All reported results are averages of five runs. The standard deviation of these five runs is, for all experiments, reported in the appendix. 1

\section{A. Rotated MNIST experiments}

For the Rotated MNIST experiments, we consider $T=20$ and $\gamma=5^{\circ}$.

As shown in Table II, CSQN outperforms EWC, MAS and LWF after 20 tasks. This is true for all four settings, between

\footnotetext{
${ }^{1}$ For our code, see github.com/StevenVdEeckt/csqn
} 
which the differences are relatively small. Nevertheless, increasing the number of components from $M=10$ to $M=20$ seems to slightly improve the performance of CSQN with BFGS and SR1. Moreover, we find CSQN with SR1 slightly outperforming CSQN with BFGS.

Figure 1 shows the average accuracy after each task is learned, from which we can see that CSQN consistently performs better than EWC, LWF and MAS.

However, while CSQN outperforms EWC, it substantially underperforms $\mathrm{KF}$, which reaches an impressive average accuracy of more than $94 \%$, which is approximately $10 \%$ and $19 \%$ higher (in relative terms) than the best CSQN setting and EWC, respectively. We note that this is consistent with [15], in which in similar experiments (on the MNIST dataset with a similar model), KF performed considerably better than EWC. KF does, however, not attain the highest backward transfer, as LWF achieves a positive backward transfer of almost $8 \%$. This means that LWF is able to improve the performance on previous tasks when learning new ones. However, LWF still reaches a relatively low average accuracy as it is unable to learn the new tasks well.

TABLE I: Average accuracy (ACC) and backward transfer (BWT) in $\%$ on the Rotated MNIST experiments with $\gamma=5^{\circ}$ and $T=20$.

\begin{tabular}{lrr}
\hline \multirow{2}{*}{ Model } & \multicolumn{2}{c}{ Rotated } \\
\cline { 2 - 3 } & ACC & \multicolumn{1}{c}{ BWT } \\
\hline Fine-Tuning & 58.66 & -41.15 \\
EWC & 79.29 & -17.12 \\
MAS & 82.06 & -12.48 \\
LWF & 73.59 & $\mathbf{7 . 9 4}$ \\
KF & $\mathbf{9 4 . 6 6}$ & -1.92 \\
\hline CSQN-S (10) & 85.49 & -8.21 \\
CSQN-S (20) & 86.22 & -7.46 \\
CSQN-B (10) & 85.37 & -8.45 \\
CSQN-B (20) & 85.96 & -7.96 \\
\hline
\end{tabular}

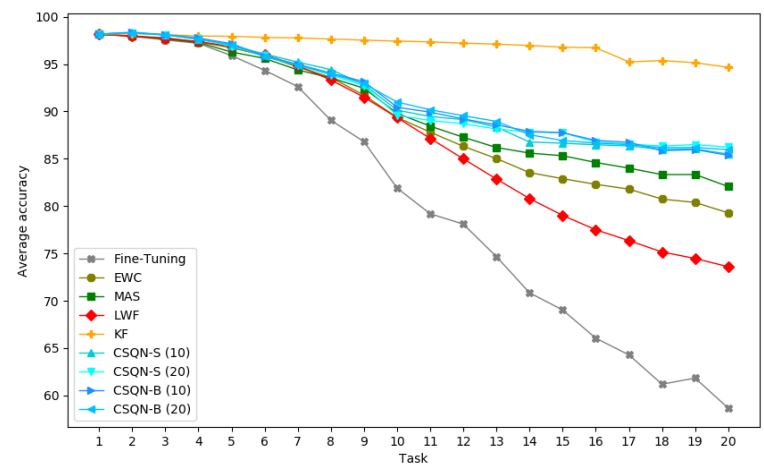

Fig. 1: Average accuracy after each task for Rotated MNIST experiments.

\section{B. Split CIFAR-10/100 experiments}

We split the CIFAR-100 dataset into $T=10$ tasks, thus obtaining a sequence of 11 tasks. Table $\mathrm{II}$ shows the final results for the Split CIFAR-10/100 experiments.

Similar conclusions can be drawn as for the Rotated MNIST experiments. CSQN substantially outperforms EWC, MAS and LWF, and this is true for all CSQN settings, except, in this case, CSQN with BFGS and $M=10$. One of the reasons for this may be that CSQN-B (10) turns out to be very sensitive w.r.t. to $\lambda$ and that none of the values of $\lambda$ that we tried worked well.

Figure 2a shows the average accuracy after training each task. Note that that until the fourth task, LWF performs better than CSQN. Nevertheless, from task 5 on, the performance of LWF drops significantly and, as a result, CSQN reaches the best performance after the 11 tasks. Similar to the Rotated MNIST experiments, we find that the performance improves when increasing the number of components from $M=10$ to $M=20$. Moreover, compared to the Rotated MNIST experiments, the increase observed here is much larger. While BFGS with $M=20$ performs better than SR1, note that in terms of storage requirements, BFGS with $M=10$ (and not BFGS with $M=20$ ) is the equivalent of SR1 with $M=20$, since for BFGS, each additional component applies a rank-2 update to the Hessian, while for SR1 this is only a rank-1 update. Considering this, SR1 seems to be preferred to BFGS. This was also true for the Rotated MNIST experiments.

In terms of backward transfer, EWC and MAS outperform CSQN. However, their average accuracy is much lower as they are unable to learn the new tasks as well as CSQN.

TABLE II: Average accuracy (ACC) and backward transfer (BWT) in \% on the Split CIFAR-10/100 and Vision Datasets experiments.

\begin{tabular}{lrrrrr}
\hline \multirow{2}{*}{ Model } & \multicolumn{2}{c}{ Split } & CIFAR & & \multicolumn{2}{c}{ Vision Datasets } \\
\cline { 2 - 3 } \cline { 6 - 7 } & ACC & BWT & & ACC & BWT \\
\hline Fine-Tuning & 18.07 & -61.07 & & 33.00 & -73.00 \\
EWC & 50.68 & -0.58 & & 69.52 & -13.98 \\
MAS & 51.21 & $\mathbf{- 0 . 1 4}$ & & 73.78 & -8.96 \\
LWF & 39.32 & -28.74 & & 75.71 & -9.44 \\
KF & & & & 77.27 & -7.53 \\
\hline CSQN-S (10) & 54.39 & -6.54 & & 78.20 & -6.70 \\
CSQN-S (20) & 55.81 & -3.82 & & $\mathbf{7 8 . 5 9}$ & $\mathbf{- 5 . 9 0}$ \\
CSQN-B (10) & 50.81 & -12.65 & & 78.31 & -6.55 \\
CSQN-B (20) & $\mathbf{5 6 . 2 9}$ & -5.36 & & 77.80 & -7.39 \\
\hline
\end{tabular}

\section{Vision Datasets}

Finally, let us turn to the Vision Datasets experiments. Table III shows the results. Once again, CSQN outperforms EWC, MAS and LWF. Compared to the Rotated MNIST experiments, CSQN now also performs better than KF. As shown by Figure $2 \mathrm{~b}$, this is true from task 2 on. Comparing the different CSQN settings, we find that increasing $M=10$ to $M=20$ results in improved average accuracy and backward transfer only for SR1, while for BFGS, the performance deteriorates, as BFGS 


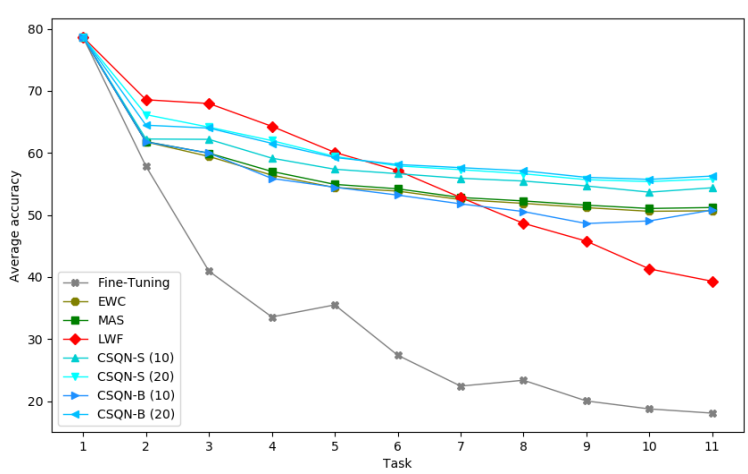

(a) Split CIFAR

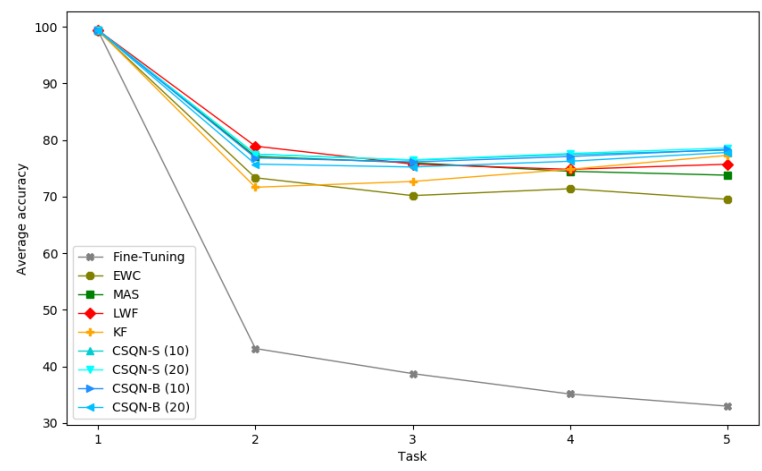

(b) Vision Datasets

Fig. 2: Average accuracy after each task for Split CIFAR and Vision Datasets experiments.

with $M=20$ reaches the lowest average accuracy of the four CSQN settings. As in the previous experiments, SR1 seems to be preferred to BFGS, though the difference between SR1 with $M=20$ and BFGS with $M=10$ is not large. Nevertheless, CSQN with SR1 and $M=20$ reaches the highest average accuracy as well as backward transfer of all methods.

\section{Limiting memory requirements}

As discussed in Section III-D, a disadvantage of CSQN are its memory requirements, which increase linearly with $T$, the number of tasks. In the same section, we proposed three strategies to overcome this linear increase. We apply them to CSQN with SR1 and $M=20$ for the Rotated MNIST and Split CIFAR-10/100 experiments. The results are shown in Table III

CT, which uses SVD to assure that memory requirements remain constant even as the number of tasks increases, suffers from severe forgetting and even performs much worse than EWC on the Split CIFAR-10/100 experiments. On the Rotated MNIST experiments, however, it performs still better than EWC, though the performance degradation with respect to the non-reduced CSQN setting is clear. CT-W, which gives $\tilde{Z}^{(\leq t)}$ a weight of $\sqrt{t}$ before it is being concatenated with $Z^{(t+1)}$ and later reduced with SVD, yields no satisfying improvement. The BTREE strategy, on the other hand, in which the $Z$ matrices of the tasks are arranged in a binary tree and reduced two-bytwo, such that any two $Z$ matrices involved in such operation represent an equal number of tasks, works much better. On the Rotated MNIST experiments, the performance degradation is, after 20 tasks, less than 1\% (in terms of average accuracy). On the Split CIFAR-10/100 experiments, the difference is larger, but not so dramatic as for the $\mathrm{CT}$ and $\mathrm{CT}-\mathrm{W}$ reduction strategies, and CSQN-S (20) with BTREE reduction strategy still outperforms EWC and MAS.

TABLE III: Average accuracy (ACC), backward transfer (BWT) and forward transfer (FWT) in \% on Rotated MNIST and Split CIFAR-10/100 experiments with different reduction strategies applied to CSQN-S (20). Last column are the memory requirements with $N$ the size of the (shared) model, $T$ the number of tasks and $M$ the number of components in BFGS and SR1.

\begin{tabular}{lrrrrrrr}
\hline \multirow{2}{*}{ Reduction } & \multicolumn{2}{c}{ Rot. MNIST } & & \multicolumn{2}{c}{ Split CIFAR } & & Memory \\
\cline { 2 - 3 } None & ACC & BWT & & ACC & BWT & & \\
CT & 86.22 & -7.46 & & 55.81 & -3.82 & \\
CT-W & 82.44 & -6.91 & & 36.84 & -16.17 & $(M \cdot T+2) N$ \\
BTREE & 82.09 & -8.49 & & 40.05 & -17.63 & \\
\hline
\end{tabular}

\section{E. Comparison between CSQN and KF}

Both CSQN and KF are based on EWC's idea to use Laplace approximation to estimate the posterior with respect to previous tasks. Since EWC uses the diagonal of the FIM in Laplace approximation, it assumes that the parameters of the model are approximately independent. CSQN and KF both try to overcome this. To this end, KF considers Kronecker-factored approximations of the FIM [16], which allows the FIM to be efficiently and compactly obtained per layer. As such, KF's Hessian approximation is block diagonal and thus considers interactions between parameters in the same layer. CSQN, on the other hand, considers the Hessian approximations obtained in QN methods. To obtain these Hessian approximations, it uses the idea of SQN methods, which suggests to sample around the current model parameters. Consequently, CSQN's Hessian approximation is no longer diagonal, and interactions between any two parameters could be considered. Another advantage of CSQN is that it is very easy to implement for any network architecture, since sampling around the current model parameters and computing $M+1$ gradients (at the current model parameters and the sampled model parameters) is all that is required to obtain the Hessian approximations. However, a disadvantage of CSQN is that the Hessian estimate is a very low rank approximation, as $M$ usually very small with respect to $N$.

In the experiments, we find that KF is superior to CSQN on the Rotated MNIST benchmark, while CSQN outperforms KF on the more challenging Vision Dataset benchmark. These results are consistent with [15], in which the difference between $\mathrm{KF}$ and EWC was much larger on the MNIST experiments using an MLP than on the Vision Datasets experiments with a LeNet-architecture. 


\section{CONCLUSION}

In this paper, we proposed CSQN, an algorithm for Continual Learning which, while using its reasoning, overcomes EWC's assumption of (approximately) independent model parameters. This is done by considering EWC's diagonal FIM an initial Hessian estimate and extending it with QN methods. Moreover, by considering SQN, the extended Hessian estimate can be obtained without using the QN methods as an optimizer. Simply sampling a number of points around the current model parameters in the parameter space and computing the gradients at these points with respect to a set of representative samples, is all that is required to improve EWC's Hessian approximation. In our experiments, we found CSQN significantly outperforming EWC as well as other baselines, including KF on the Vision Datasets experiments. Moreover, by introducing the BTREE reduction strategy, our method's main disadvantage, namely the linear increase of the memory requirements with $T$, the number of tasks, was alleviated.

\section{REFERENCES}

[1] Michael McCloskey and Neal J. Cohen. Catastrophic interference in connectionist networks: The sequential learning problem. volume 24 of Psychology of Learning and Motivation, pages 109-165. Academic Press, 1989.

[2] James Kirkpatrick, Razvan Pascanu, Neil Rabinowitz, Joel Veness, Guillaume Desjardins, Andrei A. Rusu, Kieran Milan, John Quan, Tiago Ramalho, Agnieszka Grabska-Barwinska, Demis Hassabis, Claudia Clopath, Dharshan Kumaran, and Raia Hadsell. Overcoming catastrophic forgetting in neural networks, 2017.

[3] David J. C. MacKay. A practical bayesian framework for backpropagation networks. Neural Comput., 4(3):448-472, May 1992.

[4] Jorge Nocedal and Stephen J. Wright. Numerical Optimization. Springer, New York, NY, USA, second edition, 2006.

[5] Albert S. Berahas, Majid Jahani, Peter Richtárik, and Martin Takáč. Quasi-newton methods for deep learning: Forget the past, just sample, 2020.

[6] Matthias Delange, Rahaf Aljundi, Marc Masana, Sarah Parisot, Xu Jia, Ales Leonardis, Greg Slabaugh, and Tinne Tuytelaars. A continual learning survey: Defying forgetting in classification tasks. IEEE Transactions on Pattern Analysis and Machine Intelligence, page 1-1, 2021.

[7] Friedemann Zenke, Ben Poole, and Surya Ganguli. Continual learning through synaptic intelligence, 2017.

[8] Rahaf Aljundi, Francesca Babiloni, Mohamed Elhoseiny, Marcus Rohrbach, and Tinne Tuytelaars. Memory aware synapses: Learning what (not) to forget, 2018.

[9] Sang-Woo Lee, Jin-Hwa Kim, Jaehyun Jun, Jung-Woo Ha, and ByoungTak Zhang. Overcoming catastrophic forgetting by incremental moment matching, 2018.

[10] Cuong V. Nguyen, Yingzhen Li, Thang D. Bui, and Richard E. Turner. Variational continual learning, 2018.

[11] Alexey Kutalev. Natural way to overcome the catastrophic forgetting in neural networks, 2020.

[12] Jonathan Schwarz, Jelena Luketina, Wojciech M. Czarnecki, Agnieszka Grabska-Barwinska, Yee Whye Teh, Razvan Pascanu, and Raia Hadsell. Progress \& compress: A scalable framework for continual learning, 2018.

[13] Arslan Chaudhry, Puneet K. Dokania, Thalaiyasingam Ajanthan, and Philip H. S. Torr. Riemannian walk for incremental learning: Understanding forgetting and intransigence. Lecture Notes in Computer Science, page 556-572, 2018.

[14] Xialei Liu, Marc Masana, Luis Herranz, Joost Van de Weijer, Antonio M. Lopez, and Andrew D. Bagdanov. Rotate your networks: Better weight consolidation and less catastrophic forgetting, 2018.

[15] Hippolyt Ritter, Aleksandar Botev, and David Barber. Online structured laplace approximations for overcoming catastrophic forgetting, 2018.

[16] James Martens and Roger Grosse. Optimizing neural networks with kronecker-factored approximate curvature, 2020.
[17] Geoffrey Hinton, Oriol Vinyals, and Jeff Dean. Distilling the knowledge in a neural network, 2015 .

[18] Zhizhong Li and Derek Hoiem. Learning without forgetting, 2017.

[19] Heechul Jung, Jeongwoo Ju, Minju Jung, and Junmo Kim. Lessforgetting learning in deep neural networks, 2016.

[20] Amal Rannen, Rahaf Aljundi, Matthew B. Blaschko, and Tinne Tuytelaars. Encoder based lifelong learning. 2017 IEEE International Conference on Computer Vision (ICCV), Oct 2017.

[21] Mehrdad Farajtabar, Navid Azizan, Alex Mott, and Ang Li. Orthogonal gradient descent for continual learning, 2019.

[22] Gobinda Saha, Isha Garg, and Kaushik Roy. Gradient projection memory for continual learning, 2021.

[23] Shipeng Wang, Xiaorong Li, Jian Sun, and Zongben Xu. Training networks in null space of feature covariance for continual learning, 2021.

[24] Yunfei Teng, Anna Choromanska, and Murray Campbell. Continual learning with direction-constrained optimization, 2020.

[25] Sylvestre-Alvise Rebuffi, Alexander Kolesnikov, Georg Sperl, and Christoph H. Lampert. icarl: Incremental classifier and representation learning, 2017.

[26] David Rolnick, Arun Ahuja, Jonathan Schwarz, Timothy P. Lillicrap, and Greg Wayne. Experience replay for continual learning, 2019.

[27] David Lopez-Paz and Marc'Aurelio Ranzato. Gradient episodic memory for continual learning, 2017.

[28] Davide Maltoni and Vincenzo Lomonaco. Continuous learning in singleincremental-task scenarios, 2019

[29] Arslan Chaudhry, Marc'Aurelio Ranzato, Marcus Rohrbach, and Mohamed Elhoseiny. Efficient lifelong learning with a-gem, 2019.

[30] Guannan Hu, Wu Zhang, Hu Ding, and Wenhao Zhu. Gradient episodic memory with a soft constraint for continual learning, 2020.

[31] Matthew Riemer, Ignacio Cases, Robert Ajemian, Miao Liu, Irina Rish, Yuhai Tu, and Gerald Tesauro. Learning to learn without forgetting by maximizing transfer and minimizing interference, 2019.

[32] Hanul Shin, Jung Kwon Lee, Jaehong Kim, and Jiwon Kim. Continual learning with deep generative replay, 2017.

[33] Sebastian Farquhar and Yarin Gal. A unifying bayesian view of continual learning, 2019.

[34] Ghada Sokar, Decebal Constantin Mocanu, and Mykola Pechenizkiy. Learning invariant representation for continual learning, 2021.

[35] Andrei A. Rusu, Neil C. Rabinowitz, Guillaume Desjardins, Hubert Soyer, James Kirkpatrick, Koray Kavukcuoglu, Razvan Pascanu, and Raia Hadsell. Progressive neural networks, 2016.

[36] Chrisantha Fernando, Dylan Banarse, Charles Blundell, Yori Zwols, David Ha, Andrei A. Rusu, Alexander Pritzel, and Daan Wierstra. Pathnet: Evolution channels gradient descent in super neural networks, 2017.

[37] Jaehong Yoon, Eunho Yang, Jeongtae Lee, and Sung Ju Hwang. Lifelong learning with dynamically expandable networks, 2018.

[38] Joan Serrà, Dídac Surís, Marius Miron, and Alexandros Karatzoglou. Overcoming catastrophic forgetting with hard attention to the task, 2018.

[39] Vincenzo Lomonaco and Davide Maltoni. Core50: a new dataset and benchmark for continuous object recognition. In Sergey Levine, Vincent Vanhoucke, and Ken Goldberg, editors, Proceedings of the 1st Annual Conference on Robot Learning, volume 78 of Proceedings of Machine Learning Research, pages 17-26. PMLR, 13-15 Nov 2017.

[40] Ferenc Huszár. On quadratic penalties in elastic weight consolidation, 2017.

[41] Yann LeCun and Corinna Cortes. MNIST handwritten digit database. 2010.

[42] Alex Krizhevsky. Learning multiple layers of features from tiny images. Technical report, 2009.

[43] Yuval Netzer, Tao Wang, Adam Coates, Alessandro Bissacco, Bo Wu, and Andrew Ng. Reading digits in natural images with unsupervised feature learning. NIPS, 012011.

[44] Han Xiao, Kashif Rasul, and Roland Vollgraf. Fashion-mnist: a novel image dataset for benchmarking machine learning algorithms, 2017.

[45] Yaroslav Bulatov. Notmnist dataset, 2011.

[46] Nitish Srivastava, Geoffrey Hinton, Alex Krizhevsky, Ilya Sutskever, and Ruslan Salakhutdinov. Dropout: A simple way to prevent neural networks from overfitting. J. Mach. Learn. Res., 15(1):1929-1958, January 2014.

[47] Kaiming He, Xiangyu Zhang, Shaoqing Ren, and Jian Sun. Deep residual learning for image recognition, 2015.

[48] Yerlan Idelbayev. Proper ResNet implementation for CIFAR10/CIFAR100 in PyTorch. https://github.com/akamaster/ pytorch_resnet_cifar10 Accessed: 2021-06-01. 
[49] Y. Lecun, L. Bottou, Y. Bengio, and P. Haffner. Gradient-based learning applied to document recognition. Proceedings of the IEEE, 86(11):22782324, 1998.

[50] Adam Paszke, Sam Gross, Francisco Massa, Adam Lerer, James Bradbury, Gregory Chanan, Trevor Killeen, Zeming Lin, Natalia Gimelshein, Luca Antiga, Alban Desmaison, Andreas Kopf, Edward Yang, Zachary DeVito, Martin Raison, Alykhan Tejani, Sasank Chilamkurthy, Benoit Steiner, Lu Fang, Junjie Bai, and Soumith Chintala. Pytorch: An imperative style, high-performance deep learning library. In H. Wallach, H. Larochelle, A. Beygelzimer, F. d'Alché-Buc, E. Fox, and R. Garnett, editors, Advances in Neural Information Processing Systems 32, pages 8024-8035. Curran Associates, Inc., 2019.

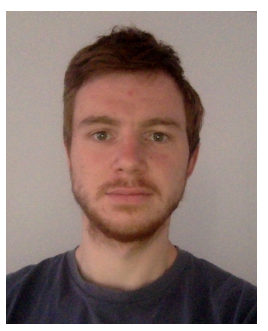

Steven Vander Eeckt Steven Vander Eeckt obtained a Master of Science in Mathematical Engineering (burgerlijk ingenieur) from the KU Leuven in 2019 and a Master of Science in Data Science, Big Data from Université libre de Bruxelles in 2020. Since September 2020, he is a PhD student at Departement of Electrical Engineering ESAT-PSI at KU Leuven. $\mathrm{His} \mathrm{PhD}$ topic is Continual Learning in Speech Recognition.

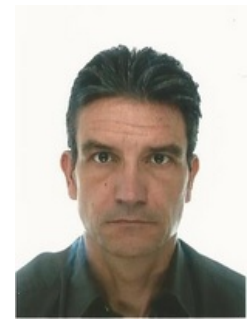

Hugo Van hamme Hugo Van Hamme (M'92-SM'11) received the master's degree in engineering (burgerlijk ingenieur) from Vrije Universiteit Brussel (VUB), Brussels, Belgium, in 1987, the M.Sc. degree from Imperial College London, London, U.K, in 1988, and the Ph.D. degree in electrical engineering from VUB, in 1992. From 1993 to 2002, he was with LH Speech Products and ScanSoft, initially as a Senior Researcher and later as a Research Manager. Since 2002, he has been a Professor with the Department of Electrical Engineering, KU Leuven, Leuven, Belgium. His main research interests include automatic speech assessment, assistive speech technology, source separation, and noise robust speech recognition, models of language acquisition, and computer-assisted learning. 